\title{
Prognostic factors in radical cystectomy affecting survival
}

\author{
Erdogan Aglamis ${ }^{1}$, Gokhan Toktas², Erdinc Unluer², Cemal Tasdemir ${ }^{3}$, Cavit Ceylan²
}

${ }^{1}$ Clinics of Urology, Elazig Education and Research Hospital, Turkey

${ }^{2}$ Clinics of Urology, Istanbul Education and Research Hospital, Istanbul, Turkey

${ }^{3}$ Inonu University Medical Faculty, Turkey

Submitted: 7 June 2011

Accepted: 2 October 2011

Arch Med Sci 2012; 8, 4: 650-654

DOI: 10.5114/aoms.2012.30288

Copyright @ 2012 Termedia \& Banach

\author{
Corresponding author: \\ Cemal Tasdemir MD \\ Department of Urology \\ Medical Faculty \\ Inonu University \\ 44280 Malatya \\ Turkey \\ Phone: 05335122377 \\ E-mail: \\ tasdemrcemal@yahoo.com
}

\begin{abstract}
Introduction: The aim of the study was to evaluate the prognostic factors in radical cystectomy affecting survival.

Material and methods: A total of 100 hundred patients were included in the study. Incontinent diversion was applied to 73 of these, and continent diversion to 27. Prospective and retrospective data of the patients were examined. The prognostic value for survival was evaluated for of lymph node involvement, tumor grade (low grade: grade 0 -II, high grade: $\geq$ III or epidermoid carcinoma), tumor stage (low stage: stage pTO-2, high stage: stage $\geq 3$ a pT3a), presence of preoperative unilateral of bilateral hydronephrosis, presence of preoperative uremia (serum urea value: $\leq 60)$, and age ( $>70$ and $\leq 70$ years of age) on survival were investigated. Kaplan-Meier survival analysis and Log-Rank statistical methods were used in the study.

Results: Grade, stage, uremia, and lymph node involvement had significant effects on survival ( $p$ values $0.0002,0.03,0.01$, and 0.02 , respectively). Presence of preoperative hydronephrosis and age had no statistically significant effects on survival ( $p$ values 0.8 and 0.2 , respectively).

Conclusions: Tumor grade, tumor stage, preoperative uremia, and lymph node involvement are prognostic factors affecting survival. Advanced age and presence of preoperative hydronephrosis have no prognostic value for survival. The presence of uremia in the preoperative assessment of the patients is more important than hydronephrosis.
\end{abstract}

Key words: bladder cancer, radical cystectomy, prognostic factor.

\section{Introduction}

Invasive bladder tumors are defined as those that range from involvement of the superficial muscle layer of the bladder (stage T2) to those at stage T4, where the tumor infiltrates the prostatic urethra in continuity. Radical cystectomy is the gold standard treatment for locally invasive bladder cancers [1, 2]. The main indications for radical cystectomy include muscle-invading bladder tumor, stage $\mathrm{T} 1$ recurring tumors with high grades and resistance to intravesical chemo-immunotherapy, widespread superficial tumors that cannot be treated with intravesical chemo-immunotherapy or transurethral resection, and in situ chemo-immunorefractory carcinoma. The main prognostic factors accepted for radical cystectomy include tumor stage, grade, multifocality, lymph node metastasis, vascular invasion and systemic spreading, performance status, systemic diseases such as cardiopulmonary diseases, anemia, and renal insufficiency, and genetic changes such as mutations in the P53 tumor suppression gene [3]. 
Knowing the prognostic factors that affect the survival and the possible survival period of invasive bladder cancer patients and the complications observed after the treatment performed will no doubt play an important role in the choice of treatment modality and determination of the operation type.

In our study, we aimed to determine the survival periods and the prognostic factors that affect the survival periods in patients who undergo radical cystectomy for bladder cancer.

\section{Material and methods}

The local ethics committee approved our study protocol. A total of 106 patients were involved in the study. All had undergone radical cystectomy because of bladder cancer within a period of 8 years. Five patients were excluded since they could not be reached in the postoperative period. A sixth patient who had bad performance status died in the perioperative period and was also left out of the study. Additional oncologic diseases were further reasons for exclusion. In total, 100 patients were included in the study. Patients were preoperatively evaluated by biochemical parameters/electrolyte analysis, lung X-rays, abdominal computed tomography, and renal ultrasonograms (USG) before radical cystectomy was performed. Patients were followed up at intervals of 3 months in the first year, at intervals of 6 months in the second and third years, and then at 1-year intervals. Lung $\mathrm{X}$-rays, related biochemical parameters (electrolytes, blood gases), urine cytology and urinary system USG were performed at all follow-up visits. Intravenous pyelograms were taken in patients with hematuria or hydronephrosis in the USG. Bone scans and computed tomography/magnetic resonance imaging were performed annually. Cytology of the irrigation fluid and urethroscopy-biopsy were performed in patients with urethrorrhagia where the incontinent diversion operation had been performed. Previous medical records of the 100 patients were examined retrospectively, and new data for routine controls were also examined prospectively. In this study, 95 of the 100 patients were male (95\%), and 5 (5\%) were female. Of the 73 patients who underwent incontinent diversion,
69 had ileal conduits, and 4 had ureterocutaneostomies. Of the 27 patients who underwent continent diversion, Hautman urinary diversion was applied to 7, Mainz-II to 3, and Studer urinary diversion to 17 (Table I). Mean hospitalization and follow-up periods, survival, and factors affecting survival were examined. We evaluated the effects of tumor stage, grade, age ( $>70$ and $\leq 70$ years of age), lymph node involvement, uremia, and presence of uni- or bilateral hydronephrosis on survival. Grade 0 and 2 were considered as low grade, and grade 3 and 4 and epidermoid carcinoma were considered as high grade. Stage TO-T2 patients were considered as low stage, and stage $\geq \mathrm{T} 3 \mathrm{a}$ were considered as high stage. Patients with serum urea levels $\geq 60 \mathrm{mg} / \mathrm{dl}$ were considered as uremic. Postoperative local recurrence, distant metastases, and urethral recurrence rates were calculated.

\section{Statistical analysis}

The data were analyzed using SPSS version 13.0 for Windows. Log-rank and Kaplan-Meier survival analysis tests were used in statistical analyses.was used ( $p \leq 0.05$ was considered significantly).

\section{Results}

The mean age of the 100 patients was $56.16 \pm 8.53$ (min: 38 - max: 72 ) years, and the mean hospitalization period was $5.19 \pm 6.44$ (min: 7 - max: 44) days. A total of 33 patients were $>70$ years of age, and 67 patients were $\leq 70$ years of age. The mean followup period was $27.7 \pm 23.6$ months. Of the 100 patients, 37 (37\%) died. Of these 37 patients, 3 had diabetes mellitus and 4 had hypertension. No other diseases were evident that would negatively affect survival.

Urethrectomy was performed upon finding urethral tumors in 5 patients (5\%) at follow-up. Local recurrence was found in 2 patients (2\%), and distant metastases were found in 8 (8\%). Patients who were classified as $>\mathrm{T} 3 \mathrm{~b}$ and/or those with lymph node involvement according to histopathological staging, or those patients with local recurrence or distant metastases in follow-up visits, were referred to oncology.

Table I. Diversion types of the 100 patients included in the study

\begin{tabular}{|llcc|}
\hline Variable & & \multicolumn{2}{c|}{ Number of patients } \\
\cline { 3 - 4 } & & $n(\%)$ & Total, $n$ (\%) \\
\hline \multirow{2}{*}{ Incontinent } & lleal conduit & $69(69)$ & $73(73)$ \\
& Ureterocutaneostomy & $4(4)$ & $27(27)$ \\
\hline \multirow{2}{*}{ Continent } & Studer & $17(17)$ & \\
& Hautman & $7(7)$ & \\
& Mainz-II & $3(3)$ & \\
\hline
\end{tabular}


Table II. Mean survival period, 3-year survival, and $p$ values in the patient group according to prognostic factors (logrank)

\begin{tabular}{|c|c|c|c|c|c|}
\hline \multicolumn{2}{|l|}{ Prognostic factor } & \multirow{2}{*}{$\begin{array}{c}\text { Patients } \\
n(\%) \\
41(41)\end{array}$} & \multirow{2}{*}{$\begin{array}{c}\begin{array}{c}\text { Mean survival } \pm \\
\text { standard deviation } \\
\text { [months] }\end{array} \\
33.3 \pm 3.6\end{array}$} & \multirow{2}{*}{$\begin{array}{c}\text { 3-year } \\
\text { survival [\%] } \\
65\end{array}$} & \multirow{2}{*}{$\begin{array}{c}\text { Value of } p \\
0.03\end{array}$} \\
\hline Stage & Low & & & & \\
\hline & High & $59(59)$ & $21.8 \pm 2.7$ & 25 & \\
\hline \multirow[t]{2}{*}{ Grade } & Low & $33(33)$ & $39.4 \pm 3.8$ & 70 & 0.0002 \\
\hline & High & $67(67)$ & $19.3 \pm 2.4$ & 23 & \\
\hline \multirow[t]{2}{*}{ Lymph node } & Negative & $85(85)$ & $28.1 \pm 2.4$ & 45 & 0.02 \\
\hline & Positive & $15(15)$ & $14.3 \pm 4.7$ & 10 & \\
\hline \multirow[t]{2}{*}{ Uremia } & No & $80(80)$ & $28.5 \pm 2.6$ & 45 & 0.01 \\
\hline & Yes & $20(20)$ & $16.5 \pm 3.4$ & 20 & \\
\hline \multirow[t]{2}{*}{ Hydronephrosis } & No & $64(64)$ & $26.8 \pm 2.7$ & 45 & 0.8 \\
\hline & Yes & $36(36)$ & $24 \pm 6.6$ & 35 & \\
\hline \multirow[t]{2}{*}{ Age } & $>70$ & $67(67)$ & $27.7 \pm 2.8$ & 43 & 0.2 \\
\hline & $\leq 70$ & $33(33)$ & $22.7 \pm 3.7$ & 35 & \\
\hline
\end{tabular}

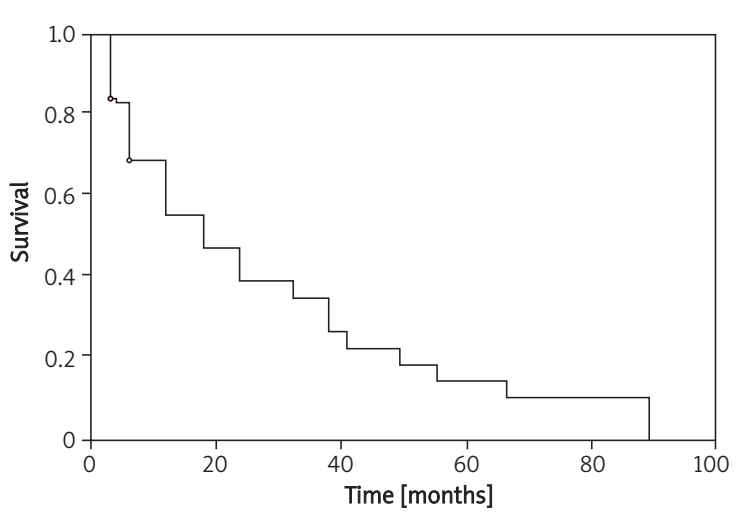

Figure 1. Survival for the entire patient group (KaplanMeier)

Analysis of prognostic factors is presented in Table II. The mean survival for the 100 patients included in the study was $26 \pm 2.2$ months. The 3-year survival was 35\% (Figure 1).

Three-year survival in low stage patients was $65 \%$ while the rate was $25 \%$ in high stage patients. The difference was statistically significant $(p=0.03$, log-rank). Similarly, three-year survival rates in low grade and high grade patients were $70 \%$ and $23 \%$, respectively. The difference was statistically significant ( $p=0.0002$, log-rank) (Table II).

Survival rate was detected to be significantly low in patients with pelvic node involvement. The threeyear survival rate was $10 \%$ and $45 \%$ in patients with and without pelvic lymph node involvement, respectively (log-rank $p=0.02$ ). The difference between 3-year survival rates of the patients with or without pre-operative unilateral or bilateral hydronephrosis was not statistically significant ( $p=0.8$, log-rank) (Table II). However, the 3-year survival rate was significantly lower in patients with serum urea level of $\geq 60 \mathrm{mg} / \mathrm{dl}$ ( $p=0.01$, log-rank) (Table II). The difference between 3-year survival rates of the patients $>70$ and $\leq 70$ years old was not statistically significant ( $p=0.2$, log-rank).

\section{Discussion}

Treatment protocols for bladder cancer are based on whether the tumor is superficial or invasive. While the first treatment for superficial bladder cancer is almost always transurethral resection, more aggressive treatment modalities are applied for invasive bladder cancers, including partial cystectomy, transurethral resection, radiotherapy, and radical cystectomy. Among these treatment alternatives, radical cystectomy is regarded as the gold standard. However, knowing the expected survival period, and the prognostic factors affecting survival, postoperative mortality and morbidity ratios, and the basal quality of life expected for the postoperative period are no doubt important in the selection of treatment alternatives and the diversion type.

Various figures have been reported for the 5-year survival after radical cystectomy performed because of invasive bladder cancer, including $68.9 \%, 51 \%$, and $46 \%$ [4-6]. Similarly, different 3-year survival rates have been reported, at $54.1 \%$ and $76.3 \%$ $[4,7]$. Tumor stage is reported as an effective prognostic factor in determining survival [8-11]. This was also the case in the present study, as increased tumor stage negatively affected prognosis for survival. The 5-year survival in the follow-up of $58 \mathrm{pa}$ tients reported by Hammar Ather et al. following radical cystectomy was $87 \%$ for PT0, $44 \%$ for PT2 and $40 \%$ for PT3 patients [10]. In another study, 5 -year survival was $78 \%$ for stage $\mathrm{T} 2,70 \%$ for T3a, $58 \%$ for T3b, and $46 \%$ for T4a patients [12]. Since the mean follow-up period for our patients was 27.7 months, the 3 -year survival was extrapolated, 
and was estimated at 35\%. Factors such as cardiopulmonary disease rate, and the presence of any other accompanying disorders such as diabetes mellitus or liver and kidney dysfunctions, as well as the experience of the surgical team and the surgical techniques used, can also be causes of variations in survival rates from those previously reported in the literature. However, the prognostic value of tumor stage is a generally accepted opinion worldwide.

Tumor grade is an important prognostic factor in patients who have undergone radical cystectomy. Survival rates for patients with high-grade tumors are poorer than for those with low grade tumors $[4,9]$. The 5 -year follow-up results of 1026 patients who underwent radical cystectomy indicated that tumor grade, pathological stage, and lymph node involvement were prognostic factors that significantly affected survival [13]. The 5-year survival in a study performed on 105 patients who underwent radical cystectomy was $85.2 \%$ for low-grade tumors, and $59.9 \%$ for high-grade tumors [4]. In another study performed on 93 patients, the 5-year survival was $72 \%$ for low grade and $44 \%$ for high-grade tumors [6]. The presence of a high-grade tumor is therefore indicated as a prognostic indicator for poor survival in patients following radical cystectomy in almost all of the studies reported in the literature. The findings of our study were in agreement, as grade was an effective prognostic indicator of survival. Patients must therefore be selected according to the biology of their tumors, and cystectomy must be suggested in the early period for low grade tumors, with additional therapies such as chemotherapy or radiotherapy proposed if required, although this remains controversial.

Regional lymph node involvement in patients scheduled to undergo radical cystectomy is also an important prognostic factor. Survival rates substantially decline in patients with lymph node involvement [7, 14-16]. In the follow-up of 90 radical cystectomy patients, the 5-year survival was $22 \%$ and $38 \%$ in patients with and without lymph node involvement, respectively [8]. In another study performed on 248 radical cystectomy patients, lymph node involvement was found in 40 patients (16\%), and the 5-year survival was 14 and $42 \%$ in groups with and without lymph node involvement, respectively [17]. These studies all indicate that lymph node involvement is not a good factor to use for prognosis. Our findings also support this, as the 3 -year survival rate was $10 \%$ in patients with lymph node involvement and $45 \%$ in those without lymph node involvement; this difference was statistically significant. Lymph node involvement in patients who undergo radical cystectomy must be considered as a predictor of a poor prognosis for survival, and additional therapies such as chemotherapy or radiotherapy must be considered. Frozen specimen results following pelvic lymphadenectomy will be important in determining the urinary diversion type to be applied.

Stöckle et al., in a study on 46 patients, showed that adjuvant chemotherapy drastically reduced the tumor reoccurrence risk in patients who had $\geq \mathrm{PT} 3 \mathrm{~b}$ and/or lymph node involvement [18]. We also referred our stage $>$ PT3b patients and/or patients with lymph node involvement to oncology.

In our study, the presence of bilateral or unilateral preoperative hydronephrosis had no statistically significant effect on survival. In contrast, an analysis of 20 patients (20\%) with preoperative uremia revealed that preoperative uremia was a factor that predicted a poor prognosis for survival [19]. A study by Bartsch et al. on 133 patients with hydronephrosis concluded that hydronephrosis could indicate a tumor in an advanced state; however, it was not an effective prognostic factor for survival. In contrast, a study by Skinner et al. on 415 patients with radical cystectomy found unilateral hydronephrosis in $22.7 \%$ of patients, and bilateral hydronephrosis in 5.3\%, and they reported the presence of hydronephrosis in patients as an indicator of poor prognosis for survival. Survival was found to be significantly reduced, particularly in cases with bilateral hydronephrosis [18]. However, serum urea levels or serum creatinine levels were not evaluated in that study. Therefore, whether the decrease in survival was truly related to hydronephrosis or was due to insufficiency of renal functions is not known. In our study, since the number of patients with serum creatinine level $>2.5 \mathrm{mg} / \mathrm{dl}$ was small (4 patients), statistical evaluation was not possible; however, uremia did have a significant effect on survival. Renal function is therefore an important criterion when determining the treatment options and selecting the operation type in patients with invasive bladder cancer. However, the presence of hydronephrosis alone is insufficient for this type of evaluation, and it would be wise to consider it together with serum urea and creatinine.

Our study indicated that age had no effect on survival. In a study performed by Wood et al., 136 patients with radical cystectomy were examined, and no differences of morbidity and mortality were seen between age groups over and under 70 years of age [19]. In another study, morbidity and mortality rates in 50 patients over 70 years of age were examined, and no differences were seen when compared to younger age groups with respect to mortality and complication rates. These authors concluded that radical cystectomy could also be safely performed on elderly patients [20]. In a study conducted on 93 patients consisting of 50 males and 43 females, gender was found to have a significant effect on survival, while age had no such effect [7]. 
We were unable to analyze the effect of gender on survival in our study, since the number of females was very small.

Age is not a criterion in bladder cancers when radical cystectomy is considered. The expected mean age differs among countries; in our study age groups were divided into groups $>70$ years of age and $\leq 70$ years of age for evaluation, and the statistical analysis indicated no effect of age on survival. According to these results, we can say that age must not be the sole criterion when assessing the patients with invasive bladder cancer for radical cystectomy and urinary diversion. The performance status of the patient and the presence of any disease that constitutes a risk for a major operation must also be taken into consideration. Radical cystectomy and urinary diversion are treatment options that can be selected safely even in elderly patients.

In conclusion, tumor grade, followed by tumor stage, preoperative uremia, and lymph node involvement, are prognostic factors that affect survival following radical cystectomy. In contrast, advanced age and the presence of preoperative hydronephrosis had no prognostic effect on survival. Determination of the presence of uremia in the preoperative assessment of the patients was more important than determination of hydronephrosis.

\section{References}

1. Saidi S, Ivanovski O, Petrovski D, et al. Lower urinary tract reconstruction following a cystectomy: experience and results in 20 patients using the "Studer" orthotopic ileal bladder substitution. Bratisl Lek Listy 2008; 109: 353-7.

2. Chłosta P, Antoniewicz AA, Szociński T, Dobruch J, Borówka A. Technique of transurethral needle core biopsy to confirm invasive bladder cancer staging. Arch Med Sci 2010; 6: 388-92.

3. Jabłonowski Z, Reszka E, Gromadzińska J, Wąsowicz W, Sosnowski M. Hypermethylation of p16 and DAPK promoter gene regions in patients with non-invasive urinary bladder cancer. Arch Med Sci 2011; 7: 512-6.

4. Kageyama S, Yoshiki T, Hamaguchi A, et al. Clinical analysis of bladder cancer patients treated by radical cystectomy. Hinyokika Kiyo 1997; 43: 1.

5. Kanamaru H, Shirahase T, Moroi S, et al. Treatment results of total cystectomy for patients with bladder cancer. Hinyokika Kiyo 1993; 39: 433.

6. May M, Helke C, Nitzke T, Vogler H, Hoschke B. Survival rates after radical cystectomy according to tumor stage of bladder carcinoma at first presentation. Urol Int 2004; 72: 103-11.

7. Waki $M$, Fukatsu $\mathrm{H}$, Nonomura $\mathrm{H}$, et al. A clinical study of total cystectomy for transitional cell carcinoma of the urinary bladder. Hinyokika Kiyo 1990; 36: 649.

8. De Geeter P, Melchior H. The use of detubularized ileum for orthotopic bladder replacement after radical cystoprostatectomy: long term results. J Urol 1993; 149: 487 A.

9. Lotan Y, Paulik VN, Rodriguez S, et al. Predictors of bladder cancer survival in a multiethnic, low socioeconomic (SES) population. J Urol 1997; 157: 256 A.
10. Hammad Ather M, Jamshaid A, Alam Z, Siddique KM, Nasir Sulaiman M. Patient's outcome of bladder cancer managed by radical cystectomy with lymphadenectomy at a university hospital. J Pak Med Assoc 2007; 57: 536-38.

11. Antunes AA, Nesrallah LJ, Dall'Oglio MF, et al. Analysis of prognostic factors in patients with transitional cell carcinoma of the bladder treated with radical cystectomy. Int Braz J Urol 2006; 32: 35-42.

12. Gupta NP, Kolla SB, Seth A, et al. Radical cystectomy for bladder cancer: a single center experience. Indian J Urol 2008; 24: 54-9.

13. El-Mekresh M, Ashamallah A, El-Attar I, El-Baz M. Radical cystectomy for carcinoma of the bladder: 1026 cases; 5 years later. J Urol 1997; 157: 374 A.

14. Stein JP, Lieskovsky G, Cote R, et al. Radical cystectomy in the treatment of invasive bladder cancer: long-term result in 1054 patients. J Clin. Oncol 2001; 19: 666-75.

15. Hara S, Miyake H, Fujisawa M, et al. Prognostic variables in patients who have undergone radical cystectomy for transitional cell carcinoma of the bladder. Jpn J Clin Oncol 2001; 31: 399-402.

16. Cheng CW, Ng CF, Chan CK, et al. A fourteen-year review of radical cystectomy for transitional cell carcinoma demonstrating the usefulness of the concept of lymph node density. Int. Braz J Urol 2006; 32: 536-49.

17. Bretheau D, Ponthieu A. Results of radical cystectomy and pelvic lynphadenectomy for bladder cancer with pelvic node metastases. Urol Int 1996; 57: 27.

18. Stöckle M, Meyenburg W, Wellek S, et al. Advanced bladder cancer (stages pT3b, pT4a, pN1 and pN2): improved survival after radical cystectomy and 3 adjuvant cycles of chemotherapy. Results of a controlled prospective study. J Urol 1992; 148: 302-7.

19. Bartsch GC, Kuefer R, Gschwend JE, et al. Hydronephrosis as a prognostic marker in bladder cancer in a cystectomyonly series. Eur Urol 2007; 51: 690-8.

20. Halebian GE, Skinner EC, Dickinson MG, et al. Hydronephrosis as a prognostic indicator in bladder cancer patients. J Urol 1998; 160: 2011.

21. Wood DP Jr, Montie JE, Maatman TJ, Beck GJ. Radical cystectomy for carcinoma of the bladder in the elderly patient. J Urol 1987; 138: 46.

22. Bassi P, Garbeglio A, Artibani W, et al. Radical cystectomy in elderly: comparative experience on so patient. J Urol 1993; 149: 498 A. 